\title{
Improvement of the College of Telecommunication and Information Network QoS by Renewing Its Basic Infrastructure
}

\author{
Dr. Ahmed A Alkhalifah ${ }^{1}$ \\ ${ }^{1}$ Assistant Professor, Computer Technology Dept. College of Telecommunication and Information, Riyadh, Saudi \\ Arabia \\ Correspondence: Dr. Ahmed A Alkhalifah, Assistant Professor, Computer Technology Dept., College of \\ Telecommunication and Information, Riyadh, Saudi Arabia
}

Received: September 7, 2018

Accepted: October 3, 2018

Online Published: October 10, 2018

doi:10.5430/ijhe.v7n5p138

URL: https://doi.org/10.5430/ijhe.v7n5p138

\begin{abstract}
College of Telecommunication and Information (CTI) provides a highly technically trained cadre that would be able to find the best scientific solutions to the problems facing us and support the development process and capable of keeping pace with the fast-global technological development (College of Telecom \& Information in Riyadh 2010). With the increasing utilization of the CTI network and an application-based usage steadily growth, several deficiencies have been encountered such as network saturation, high bandwidth consumption. The current network is unable to support the new changes required by the IT rapid development and changes. This project covers the usage of some analytical tools used to monitor network status and to make some changes and development on the CTI local network to solve some difficulties based on the data provided by those analytical tools.
\end{abstract}

Keywords: college of telecommunication and information (CTI), NMS (Network Management System), network architecture, cisco core switches, nagios, cacti

\section{Introduction}

College of Telecommunication and Information (CTI) systems experience arbitrary challenges relating to downtime and degradation of performance which significantly affect quality of services provided to employees and students within the education environment. To ensure effective operations of advanced IT architectures, CTI network monitoring services which comprehensively addresses problems to maintain high system functionality and stability is required. A network monitoring system ensures that persistent system availability, cuts down on Data Center overheads in addition to cost and resource efficiency.

This paper is divided into two parts. We describe in the first part the global analytic that we have made for the CTI network by using monitoring software such as Nagios and Cacti. In the second part, we describe different changes made on the network such as the installation of new core switches and new optical fiber links. In the following, we describe the uses of different monitoring tools to determine CTI network defects.

\section{Monitoring of the CTI network by using Nagios and Cacti}

Nagios refers to a comprehensive network and infrastructure monitoring suite which can offer a variety of network services. It can monitor almost everything within a network (Barth, 2008). This includes addressing problems ranging from server and workstation connectivity in addition to conducting individual monitoring on servers through the use of applications within both the Windows and Linux environments. Similarly, Nagios delivers unparalleled capacity planning through it automated and integrated trending in addition to capacity graphs. These features allow users to make more informed decisions by providing the requisite information for planning upgrades. Data on Nagios can be accessed real-time in formats such as digital gauge, graphing charts which are useful for managerial use (Barth, 2008). On the other Cacti was originally envisioned as geographical logging tool which sought to gather data on network devices leveraging on the RRD tool basic designed (Haiyan, 2004). However, in recent years, Cacti are made significant improvements on its user interface to brand itself as legitimate open-source monitoring project which progressively continues to introduce new features. In retrospect, Nagios is considered to demonstrate efficiency in systems and resources monitoring due to its powerful Nagios Core (Barth, 2008). In the context of Cacti, it integrates built-in SNMP support which incorporates both php-snmp, ucd-snmp and net-snmp in gathering network information. This implies that individuals with advanced scripting skills can use Cacti to collect more SNMP data 
from the network.

This part of the study will be structured into three sections; the first section offers an overview of CTI network technology SNMP based network management. The subsequent second section evaluates the design of common CTI MIB. It also highlights the design and implementation of our CTI NMS. In the third section, the focus will be on the experimental result on the small CTI testbed.

\subsection{Overview of CTI Network}

\subsubsection{Infrastructure}

The CTI mapping represents a corporate campus network which is designed to connect buildings including administrative, academic, college libraries, student centers, residence halls, gymnasiums, and other outlying structures, like conference centers, technology centers, and training institutes. CTI networks are normally interconnected with high-speed Ethernet links operating over optical fiber such as Gigabit Ethernet as shown in Figure (1).

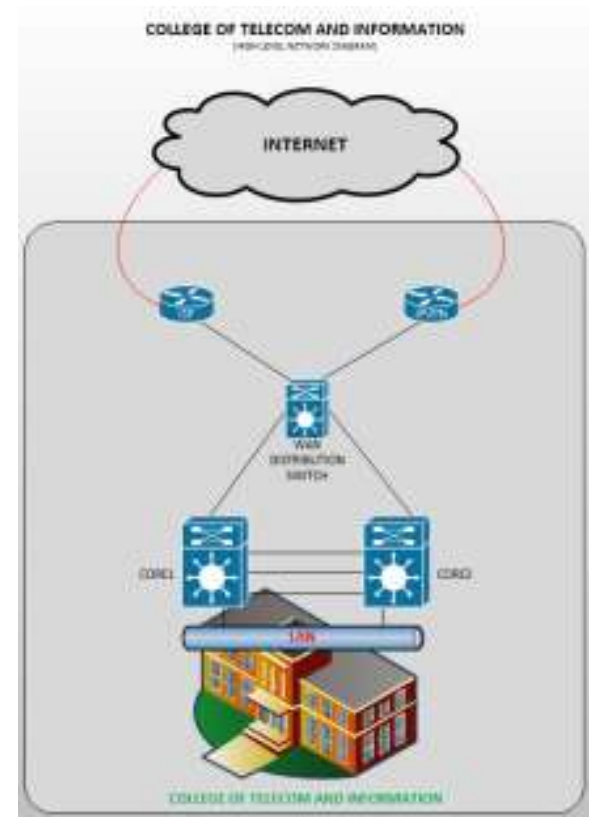

Figure 1. Overview of CTI Network Diagram

Many tools have emerged to aid in performance monitoring of networks. In recent years, there has been emergence of many system tools designed to monitor performance of networks. Most of these tools are conventionally based on the Simple Network Management Protocol (SNMP) (William stalling, 2002) (Harrington, Presuhn, Wijnen, 2002) a protocol used on IP networks for sending and transmission of information relating network performance. Other varieties of tools in monitoring network performance include packet sniffers, flow monitors, and application monitors. Some of the prominent examples of these tools include Solar Wind's Orion SNMP monitoring platform, WireShark packet capture tool, Web metrics' Global Watch, and Cisco's NetFlow flow monitoring tools.

Currently, CTI network is composed of the following infrastructure devices:

1) Cisco 4500 series switches (Core).

2) Cisco 3500 series switches (Distribution).

3) Cisco 2900 series switches (Access)

4) HP 1810 switches (Access)

5) Cisco 1900 series router (ISP and IPVPN)

6) Cisco 2800 series router (Voice Gateway)

7) Huawei VDI (Virtual Desktop Infrastructure System)

8) Windows 2012 Server (CCTV System)

9) Windows 2008 Server (Domain Controller) 
10) Windows 2000 Server (Call Manager)

11) Aruba Network (Access Point)

Until now, network devices such as Cisco switches include defined CTI MIBs in addition to a SNMP agent applied on those devices, other devices are currently focused on defining CTI MIBs on their respective SNMP supported version. Typical CTI networks will probably incorporate CTI devices from more than one vendor probably developed utilizing different SNMP version. However, for the SNMP manager to manage these hybrid devices, a common and standard MIB which defined is require in addition to implementation in all of these devices.

The current work consists on defining a common CTI MIB. Also, a CTI network Management System was developed using Cacti and Nagios which is based on common CTI MIB. The system facilitates the detection of CTI faults, recovery, network configuration, network performance monitoring by network administrators.

In the next section, we propose a free and open source application which also focuses on system and network performance which are Cacti and Nagios.

\subsubsection{CTI Network Management}

Currently, CTI is using the secured version of SNMP which is the Version 3. The two applications that monitor the system or network of CTI now are CACTI and Nagios. The following is a brief description of each one of them and how it was sued in CTI.

- Cacti is an open source web-based network and system monitoring graphing solution for IT infrastructure management. Cacti allow an administrator to poll services at regular intervals to create graphs on the resulting data. Commonly, it is used to graph time-series data on metrics such as network bandwidth utilization, running processes, CPU-Memory-Disk usage (Urban, 2017).

- Nagios is a freeware computer-software used for systems, networks and infrastructure monitoring, Nagios provides monitoring and alert services for servers, switches, applications, and services. It alerts users on system faults, and subsequent alert after the problem has been solved (Michael Guthrie Instant Nagios Starter May, 2013).

\subsection{Design and Implementation}

\subsubsection{Design}

We designed our CTI-NetMon to support the CTI network as demonstrated in Figure 2. It does not represent a generic topology, but serves as an example of the CTI network, which we would like to manage using the system proposed. Our main interest has been to manage the CTI devices utilizing SNMP. The system is designed to manage the faults, performance, and the main NMS features configuration.

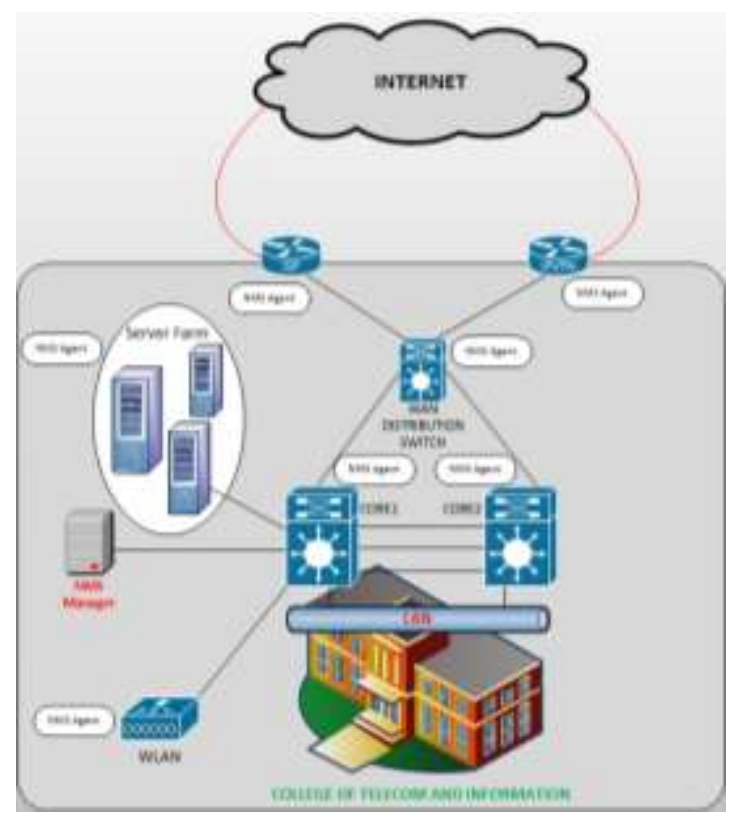

Figure 2. Proposed CTI Network Architecture 


\subsubsection{Implementation}

We have established our CTI-NetMon part using LAMP (Linux-Apache-MySQL-PHP) solution. SNMP version 3 has been chosen to add cryptographic security (Gerner, Naramore, Owens, Warden, 2005). The following packages were installed on the POC (Proof of Concept) machine.

- Apache: A Web server to display network graphs created by PHP and RRD Tool.

- MySQL: A Database server to store cacti information.

- PHP: A script module to create graphs using RRD Tool.

- PHP-SNMP: A PHP extension for SNMP to access data.

- NET-SNMP: A SNMP (Simple Network Management Protocol) is used to manage the network.

- RRD Tool: A database tool to manage and retrieve time series data like CPU load, Network Bandwidth etc.

\subsubsection{Experiment}

\subsubsection{Experiment Environment Setup}

We have made the SNMP Manager on POC (Proof of Concept) machine as described in Figure 3. We have installed a Cacti Version 0.8.8h and Nagios Core Version 4.1.1.

\begin{tabular}{|c|c|c|}
\hline \multirow{4}{*}{ Manager } & OS & CentOS 7 \\
\cline { 2 - 3 } & Memory & $8 \mathrm{Gig}$ \\
\cline { 2 - 3 } & $\mathrm{CPU}$ & Intel Core i3 @ 3.30GHz \\
\cline { 2 - 3 } & $\mathrm{HDD}$ & $500 \mathrm{Gig}$ \\
\hline \multicolumn{3}{|c|}{} \\
\hline Agent & SNMP & Version 3 \\
\hline
\end{tabular}

Figure 3. Proof of Concept of CTI-NetMon

The NMS Agent was configured on network switches using SNMP version 3 as shown in Figure 4.

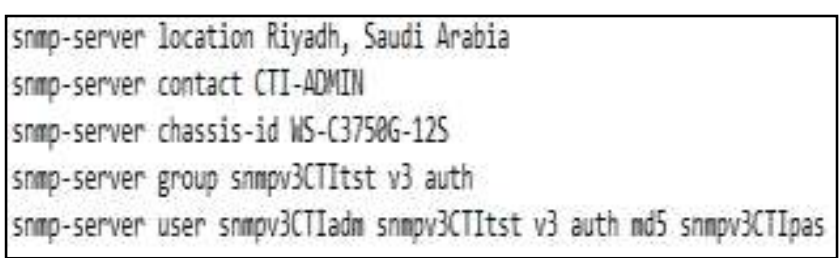

Figure 4. Configuration example on network switches

\subsubsection{Experiment Result}

To be more proactive on our daily routine, we put a four-big screen (43 inches TV) on our location with the speaker to hear the alarm if there is a down device or services that are having an issue. Figure 5 and Figure 6 shows the sample Tactical Overview of Nagios Core and Nagios XI. Figure 7 shows the preview of Graphs plugin which allows us to view the actual inbound and outbound traffic utilization. 


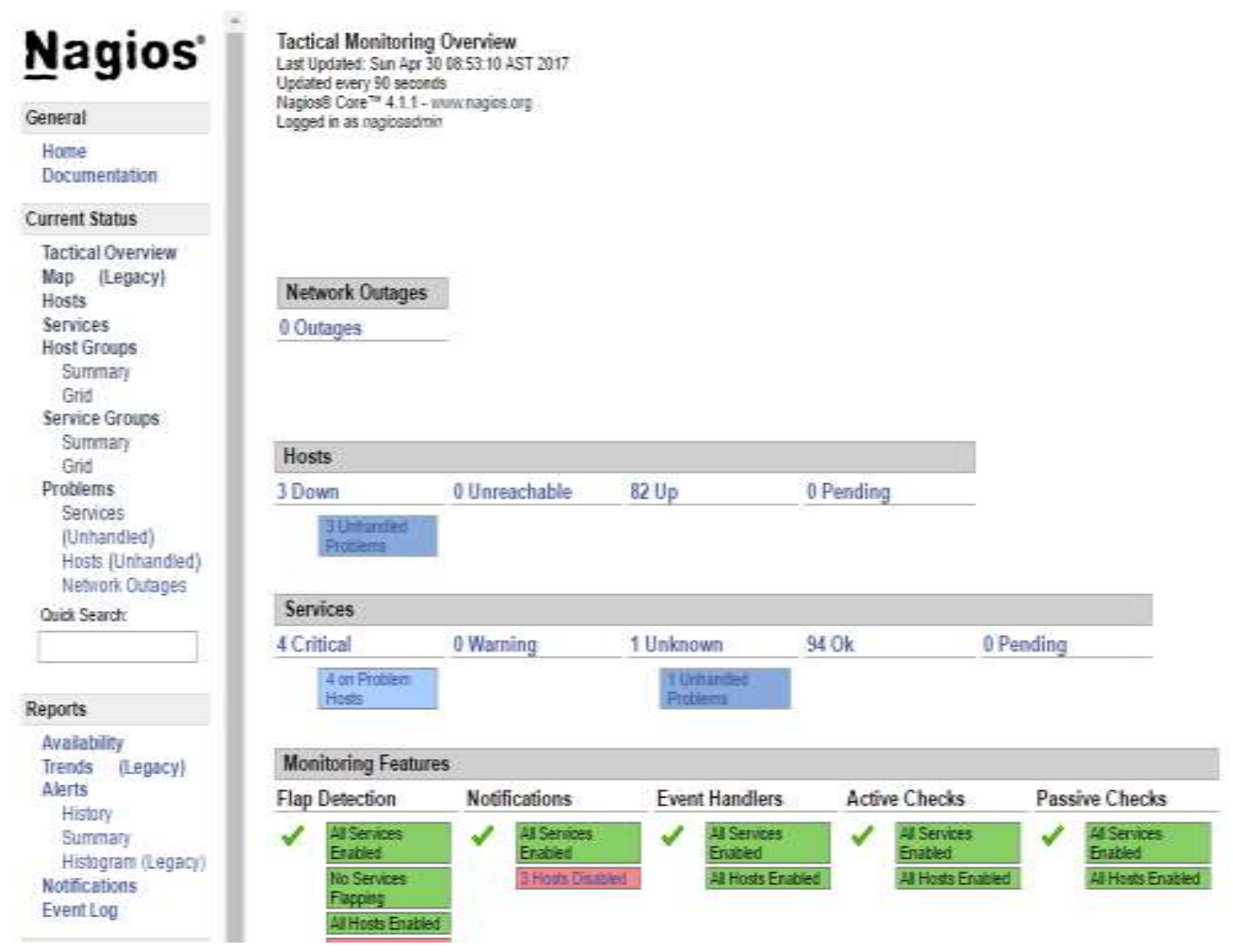

Figure 5. Main page of Nagios

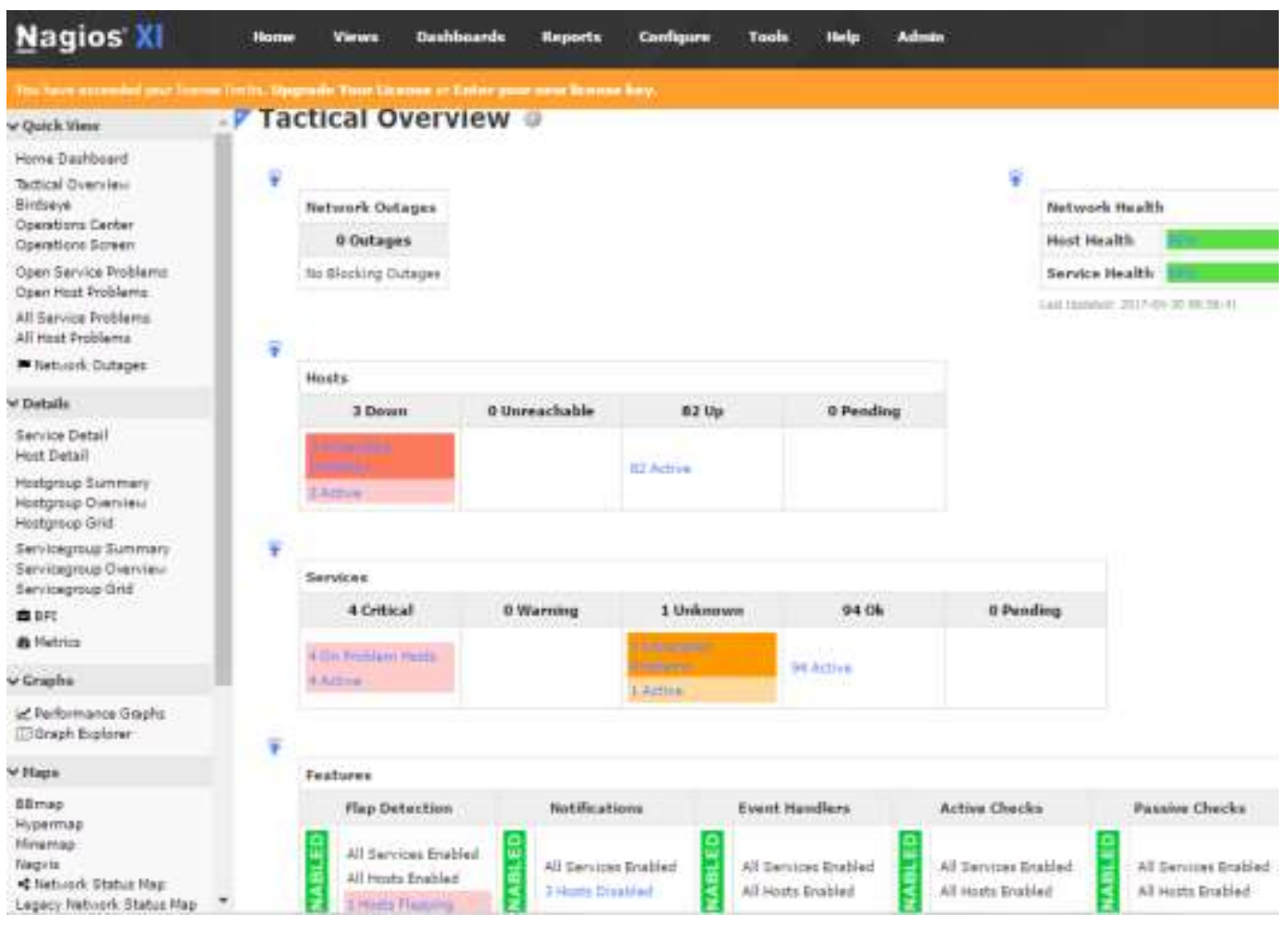

Figure 6. Tactical overview 


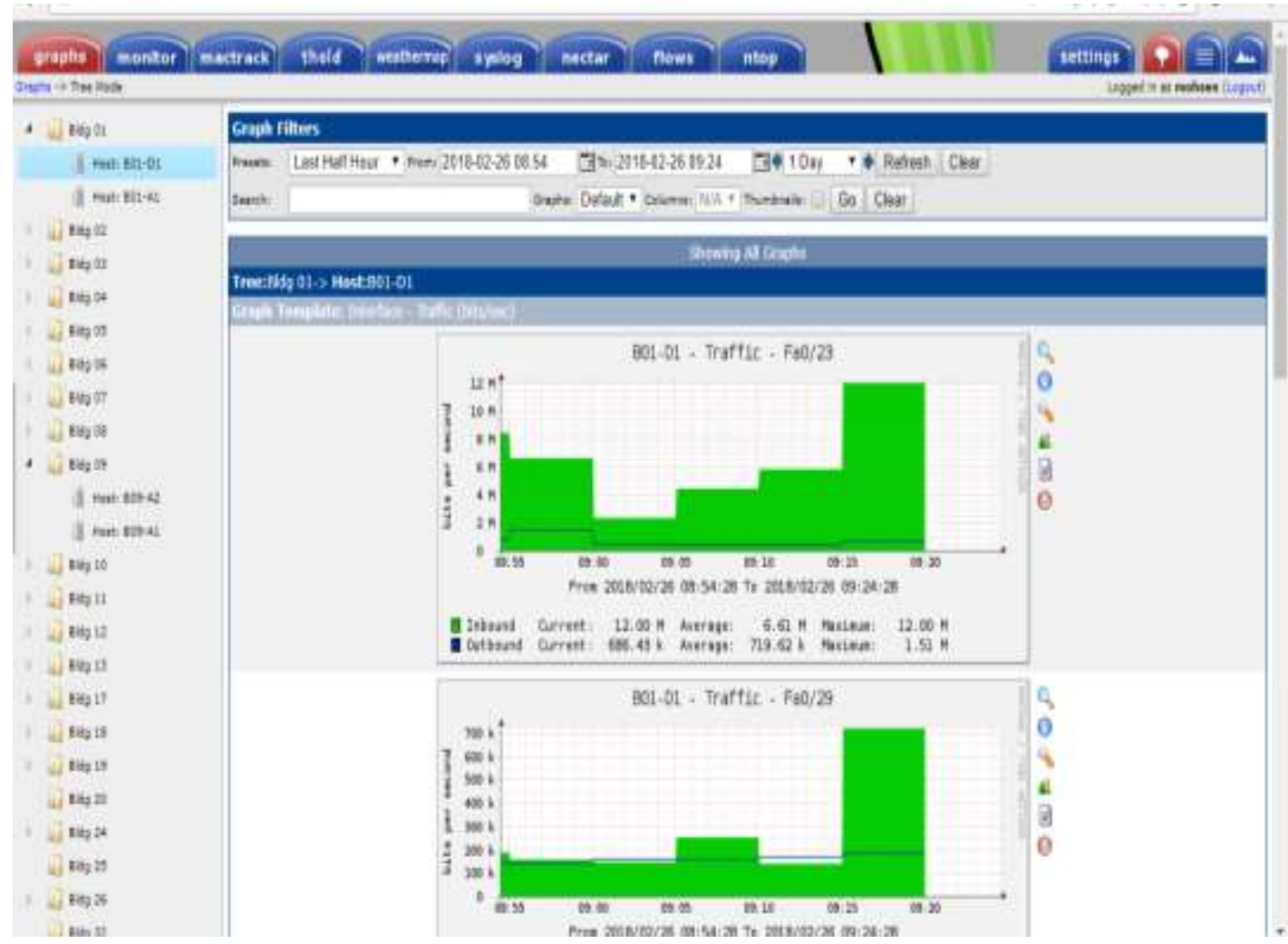

\section{CTI Infrastructure Renew}

Figure 7. Traffic in different buildings

This part is structured into two main sections. The first section highlights the overview of the new consideration such as replacing the core switches. Section II presents the new design of the fundamental transmission cables.

\subsection{Deploy New Core Switches}

CTI network topology is based on traditional data center network infrastructure. The data center networking infrastructure is designed to ensure that multiple data center tenants with different varieties of workloads can be accommodated. The coordination between the servers and clients within the network needs data center networking for the resources. The infrastructure consists of a 3-layer hierarchical model. It entails the core layer switches which link to distribution layer switches, which subsequently links to access layer switches. 


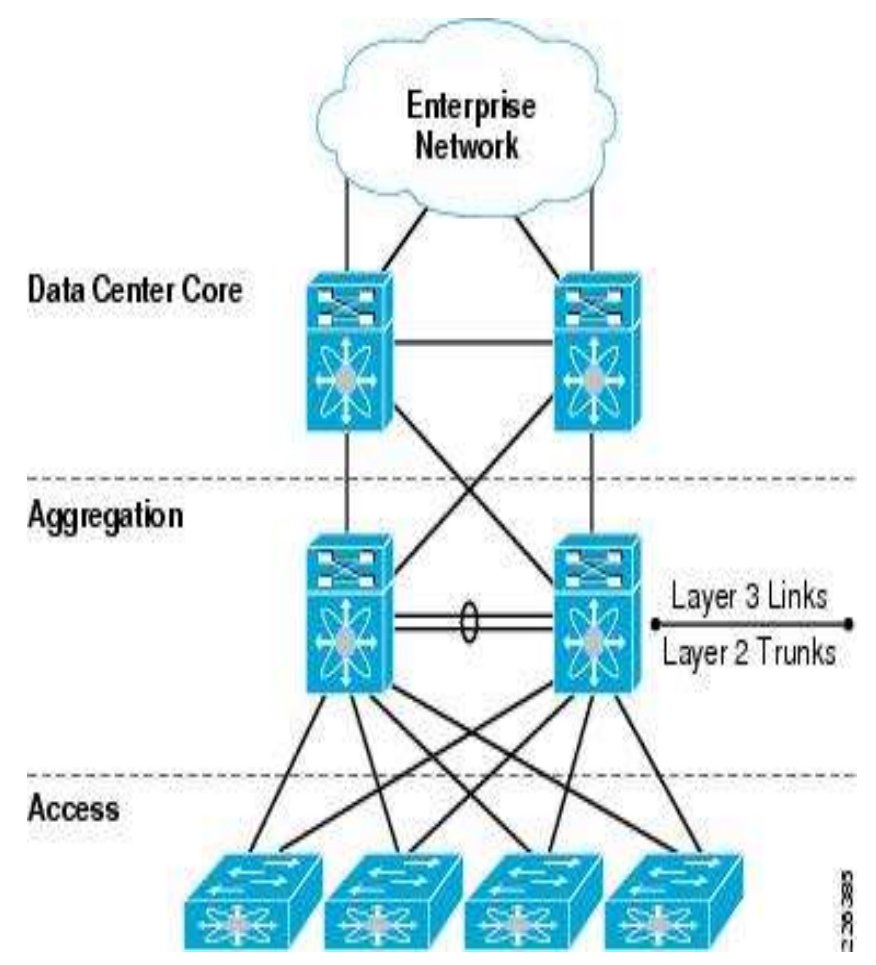

Figure 8. Basic Network Technology

Using our monitoring tools and experimental tests were done by our network managers and described previously, we noticed that the previous infrastructure suffers from:

1. Network Congestion making a traffic slow-down

2. Massive use of bandwidth even outside of peak working hours

3. Loss of the internal connection several times

4. Problems of data storage and data recovery

5. Defeat of some servers without a clear cause

6. Weakness of the internet connection and sometimes total disconnection

7. Difficulties in the integration of new applications

8. Difficulties in connecting virtual machines such as VDI (Virtual Desktop Infrastructure)

Figure 9 represents the basic infrastructure of the CTI. Given the results obtained before, we decided to change a large part of our network. We started in the first phase by changing the architectural levels like the distribution of the main devices (servers, routers, and switches) and in the second phase, we changed completely the infrastructure of our network by replacing the old cables with new optical fiber. 


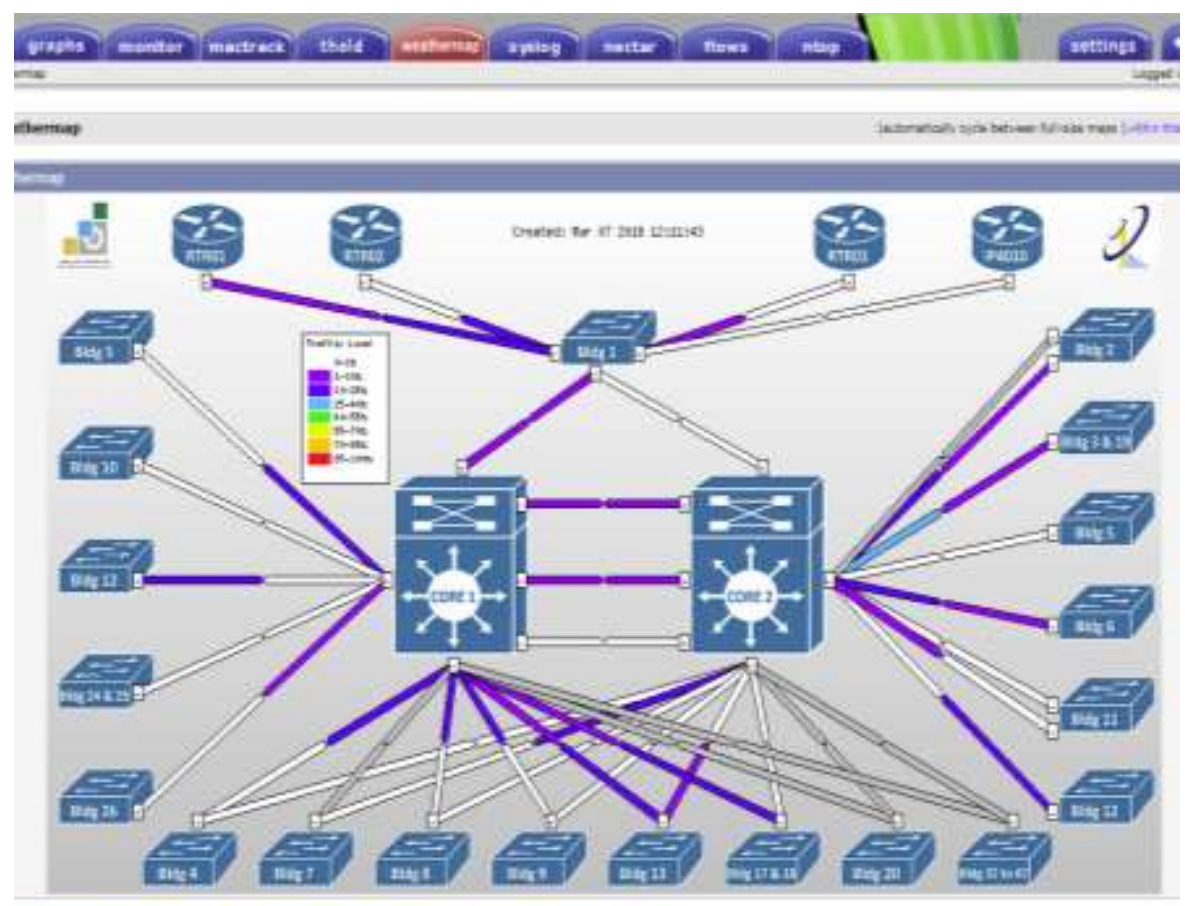

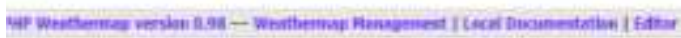

Figure 9. basic CTI network infrastructure

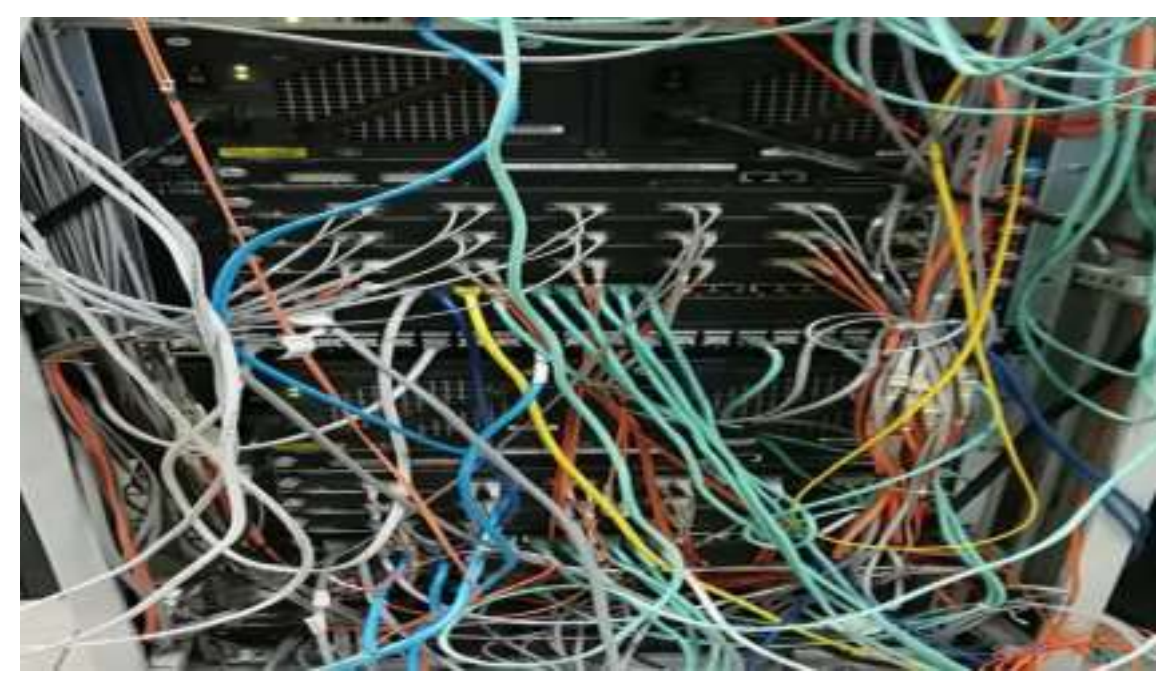

Figure 10. Old core switches 


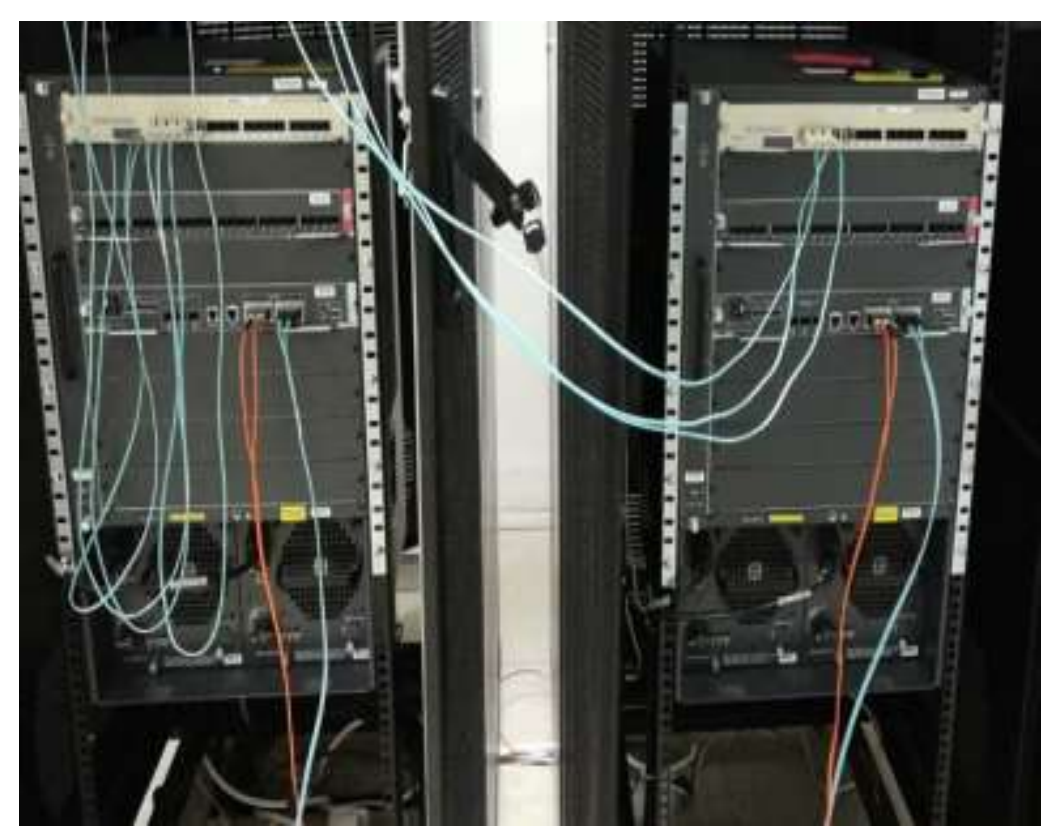

Figure 11. New core switches

Features of the new core switches (Cisco):

Cisco Catalyst 6509-E Switch

- 9 slots, 14 RU

- 32 x $40 \mathrm{GE}$ ports

- $130 \times 10 \mathrm{GE}$ ports

- $387 \times 1 \mathrm{GE}$ ports

- 385 x 10/100/1000 ports

- $\quad$ Forwarding capacity up to $510 \mathrm{Mpps}$

\subsection{Deploy New Fiber Infrastructure}

In this part, we describe the architecture of the optical fibers and the different links between the main equipment. Figure 12. represents the old cabling infrastructure in different floors. 


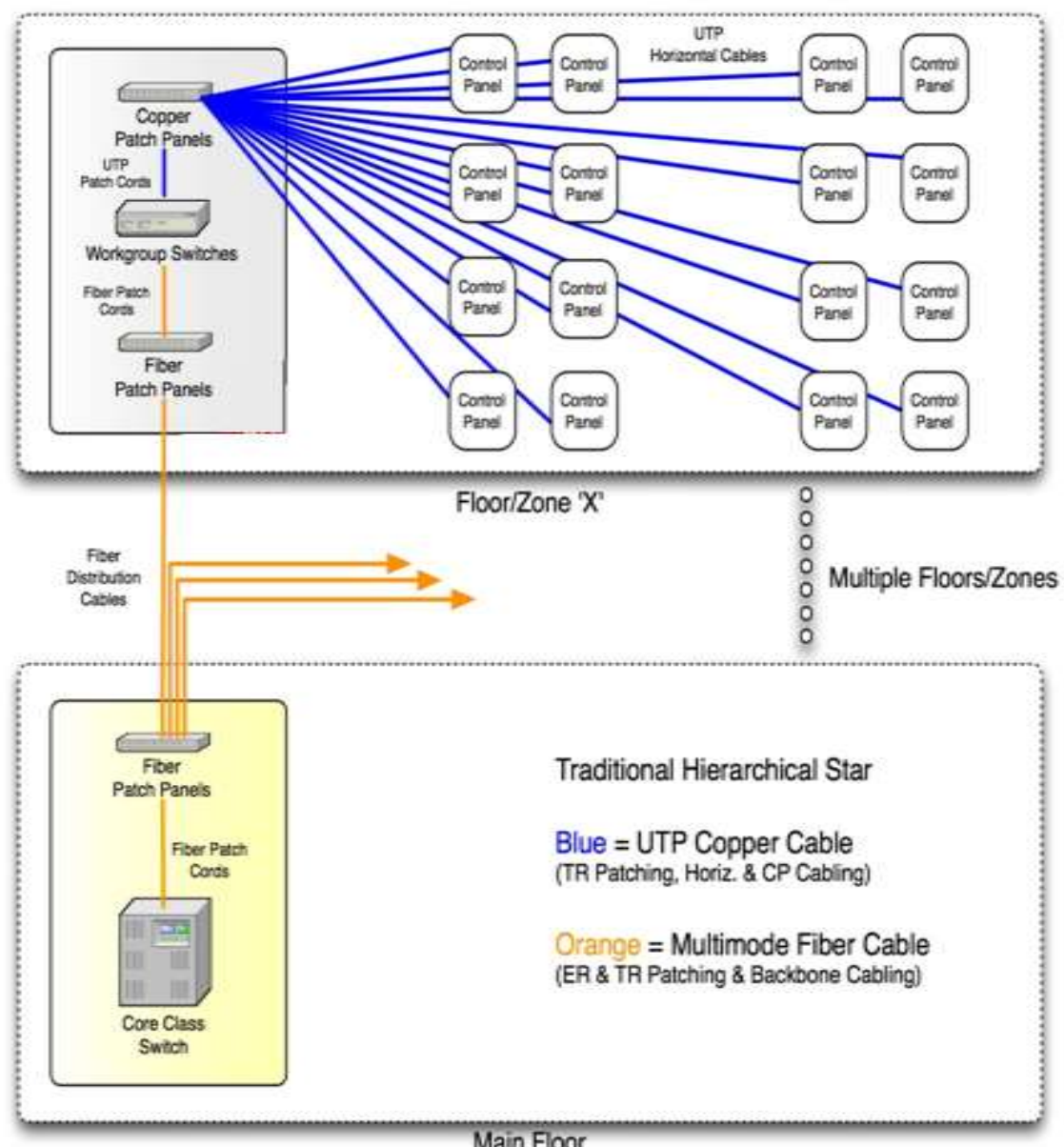

Figure 12. Traditional hierarchical topologies

To work in a deterministic order during various types of planned and unplanned outages, our network must simplify operations, optimize application performance and build resilience. The purpose of this task is to limit the effort on concentrating a solid foundation and a solid infrastructure between the accesses, distribution and core systems of our campus. It must certainly cover all the recommendations to be applied to different types of platforms according to their roles in the network.

We began the planning process by segmenting devices and equipment into hierarchical functions. This identifies the type of operations and defines logical plant network segmentation for each level Figure 13. 


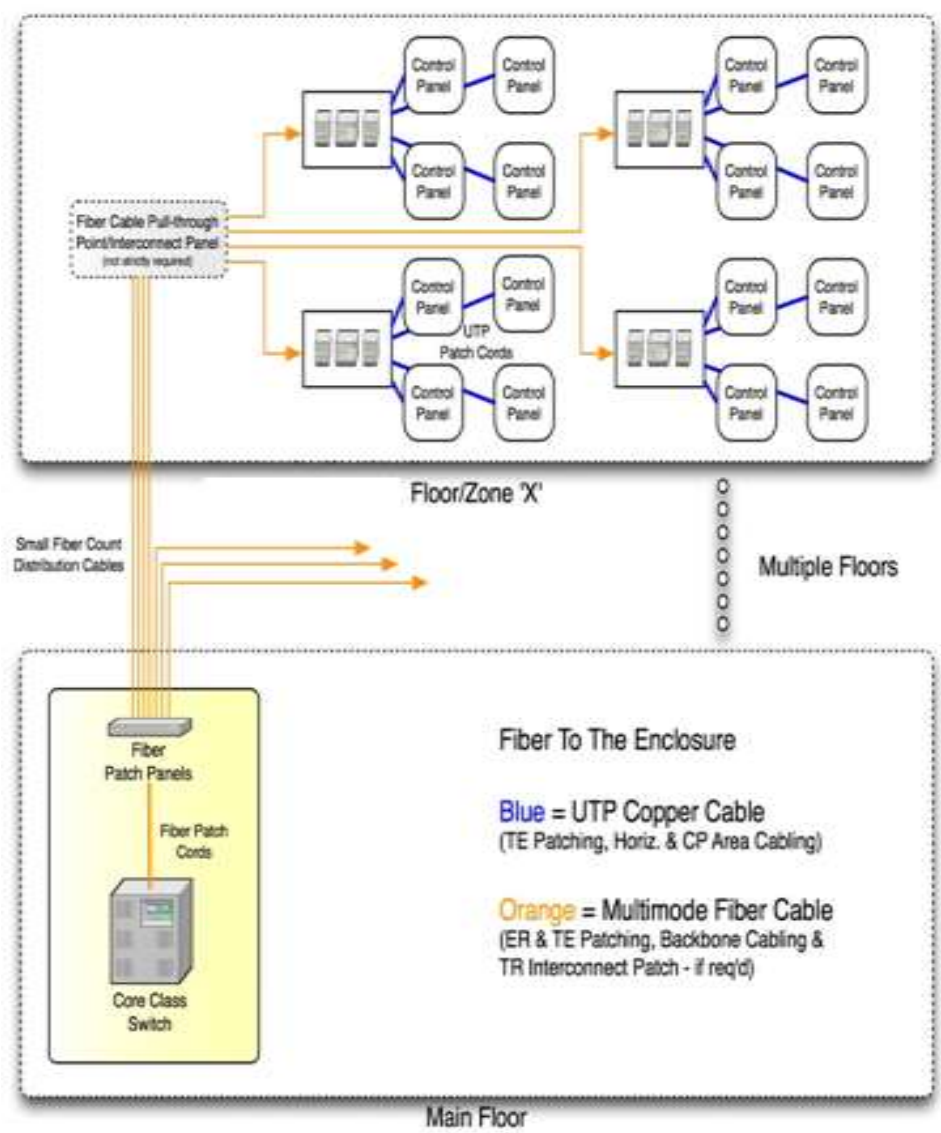

Figure 13. New hierarchical topology

The concentration of fibers in star topology reduces their used lengths during the installation. Further, it lowers the chances of network failure through connecting all systems to a central node. The typology is often considered beneficial because it is relatively easy to manage, install, configure and fault detection and removal of parts. The use of a central node in the star typology creates dependency and the risk to failure of the whole network where the central node is disabled. Additionally, other problems appear such as the degradation of the transmission quality and the difficulties of the interventions. The new fiber distribution consists of dividing the overall architecture of the fibers into small pieces giving the hand to a manipulation and administration of the smaller parts which are easier to manage. The following is a comparison table between before and after.

Table 1. Comparison between before and after

\begin{tabular}{l|lll}
\hline & Before & After \\
\hline Net speed (Mbit/s) & 12 & 54 \\
Downtime number & $\begin{array}{l}\text { Once per } \\
\text { month }\end{array}$ & No downtime \\
$\begin{array}{l}\text { Switches } \\
\text { congestion }\end{array}$ & $\begin{array}{l}\text { twice per } \\
\text { month }\end{array}$ & No congestion \\
\hline
\end{tabular}

In research, it was established that despite the positive results of the NetMon, the findings are limited to networks similar to the same require requirements we used. There is need to for further research to establish the scalability of the system in the context of large network systems. Based on the available data from the experiments in the current study, it is not possible for the researcher to project the efficiency of the system on much larger scale.

\section{Conclusion}

In summary, we concisely introduce in a first part the CTI infrastructure and encouraged the importance of the CTI network Management System. In the paper, we demonstrated the design of the common architecture of 
SNMP-based network management, design and implementation of CTI NMS called NetMon for the hybrid CTI networks. We undertook installation of Cacti and Nagios to test the system that was proposed on the POC machine where we installed CTI NetMon for ourselves and presented the test result on the network management section of this paper. In a second part, we have described the steps taken to improve our network infrastructure, in fact we have tried to sweep the limits and drawbacks of our network infrastructure by replacing the distribution of the main device like core switches and also by adopting a new architecture, which consisted of dividing the network into small cells and completely changing the transmission media with more consistent optic fibers.

This work is a description of a set of steps and decisions we took to improve the performance of Our network. This work could serve as a manual for other institutions that want to improve their networks. However, there should be some limitations to be taken in consideration including the network size and the intended user capacity. As it was described before, the project was done in an educational institute which usually has high volume demand on network services in certain times during the day, which might not be the case in other situations.

\section{Acknowledgements}

This work was supported by General Administration for Research and Studies in TVTC Corporation, member of Research Team - Dr. Mohsen DENDEN and by the System/Network Engineering Team support program supervised by the Head- Engr. Fahad Aljomah.

\section{References}

Aljomah, Fahad. (2017). Exploring the Use of Network Management System By Cacti \& Nagios Software In College Campus. Proceedings of 73rd ISERD International Conference, Bali, Indonesia, 13th -14th May 2017, ISBN: 978-93-86291-88-2.

Barth W. (2018). Nagios: System and network monitoring. No Starch Press; 2008 Oct 15.

College of Telecom \& Information in Riyadh: Brief Introduction Retrieved from: https://www.youtube.com/watch?v=5p0UAUlfTwA

Guthrie, Michael. (2013). Instant Nagios Starter. Packt Publishing Ltd, 2013.

Gerner J, Naramore E, Owens M, Warden M. (2015). Professional LAMP: Linux, Apache, MySQL and PHP5 Web Development. John Wiley \& Sons; 2005 Dec 13.

Harrington D, Presuhn R, Wijnen B. (2002). An architecture for describing simple network management protocol (SNMP) management frameworks. 2002.

Haiyan LY. (2008). The application of cacti in the campus network traffic monitoring. Computer \& Telecommunication.

Urban T. (2017). Cacti Beginner's Guide: Leverage Cacti to design a robust network operations center. Packt Publishing Ltd; 2017 Dec 27.

William Stalling SNMP, SNMPv2, SNMPv3, and RMON 1\&2, 3e 2002. 